\title{
O estresse do trabalhador de enfermagem: estudo em diferentes unidades de um hospital universitário
}

\author{
The stress of nursing workers: study in different units of a university hospital
El estrés de trabajadores de enfermería: estudio en diferentes unidades de un hospital universitario

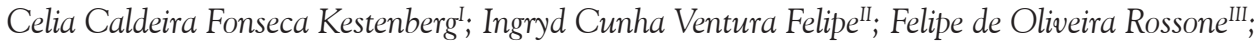 \\ Livia Moreira Delphim ${ }^{I V}$; Michele Costa Teotonio ${ }^{V}$
}

\begin{abstract}
RESUMO: Este estudo objetivou discutir o nível de estresse oriundo do trabalho dos profissionais de enfermagem de três unidades de um hospital universitário no Rio de Janeiro. Realizado de janeiro a dezembro de 2011, trata-se de uma pesquisa descritiva, com abordagem quantitativa. Participaram 85 profissionais do ambulatório central, do centro de tratamento intensivo geral e de duas enfermarias de clínica médica do hospital. A avaliação do estresse foi realizada através do Inventário de Sintomas de Stress de Lipp (ISSL) e a mensuração da exposição aos fatores estressores do ambiente de trabalho, através do Inventário de Avaliação de Fatores Estressores Subjetivos (IAFES), construído para este estudo. Constatou-se que 56,5\% dos participantes apresentavam estresse e 49,4\% encontravam-se na fase de resistência. Identificou-se que 68,5\% dos participantes apresentaram média a alta exposição aos fatores estressores laborais. Percebeu-se a necessidade de ações que visem reduzir fatores passíveis de intervenção, além de pesquisas que abordem esta temática.
\end{abstract}

Palavras-Chave: Equipe de enfermagem; estresse fisiológico; estresse psicológico; saúde do trabalhador.

\begin{abstract}
This descriptive research, with quantitative approach aims at discussing the stress level in three nursing teams' from a university hospital in Rio de Janeiro, RJ, Brazil. Eighty-five professionals were part of the study, conducted from January to December, 2011. The nurses were staffed at the central clinic, at the general intensive care unit, and at two wards in the hospital's medical clinic. The stress evaluation was held by Lipp's Inventory of Stress Symptoms (ISSL) and the measurement of the exposure to labor's environment stress factors through Inventory of Stressors Subjective Evaluation of Factors (IAFES). 56.5\% (fifty-six point five percent) of the participants were found to be stressed and $49.4 \%$ (forty-nine point four percent) were in resistance phase. $68.5 \%$ (sixty-eight point five percent) of the participants were found to be exposed to medium or high levels of labor stress factors. The need for actions seeking the decrease in factors subject to labor intervention as well as the need for research approaching the theme was identified. Keywords: Nursing team; physiological stress; psychological stress; occupational health.
\end{abstract}

RESUMEN: Este estudio tuvo por objetivo discutir el nivel de estrés provocado por el trabajo de los profesionales de enfermería de tres unidades de un hospital universitario de Rio de Janeiro - Brasil. Realizado de enero a diciembre de 2011, se trata de una investigación descriptiva, con enfoque cuantitativo. Participaron 85 profesionales del ambulatorio central, del centro de tratamento intensivo general y de dos enfermarías de clínica medica del hospital. La evaluación del estrés se realizó mediante el Inventario de Síntomas de Estrés de Lipp (ISEL) y la medición de la exposición a los factores de estrés en el entorno de trabajo, a través del Inventario de Evaluación de Factores - Estresores Subjetivos (IEFES) construido para este estudio. Se encontró que $56,5 \%$ de los participantes tenían estrés y 49,4\% estaban en fase de resistencia. Se encontró que 68,5\% de los participantes tenían entre moderada y alta exposición a los factores estresantes. Se percibió la necesidad de acciones dirigidas a reducir los factores susceptibles de intervención, y de más investigaciones que se ocupen de esta cuestión.

Palabras Clave: Equipo de enfermería; estrés fisiológico; estrés psicológico; salud del trabajador.

\section{INTRODUÇÃO}

Estresse é um assunto de larga abrangência que possui alguns conceitos e derivações. Amplamente debatido em temas de discussão popular, o assunto passou a ocupar lugar de destaque nos meios de comunicação, tornando-se objeto de interesse de pesquisadores em diversas áreas ${ }^{1,2}$.

\footnotetext{
IEnfermeira e Psicóloga Clínica. Doutora em Psicologia Social. Professora Adjunta da Faculdade de Enfermagem e Pós-Graduação Lato Sensu de Enfermagem da Universidade do Estado do Rio de Janeiro. Brasil E-mail: celiaprofuerj@gmail.com.

IIDoutora em Enfermagem pela Universidade do Estado do Rio de Janeiro. Especialista em Enfermagem do Trabalho pela Universidade Gama Filho. Enfermeira do Trabalho, Farmanguinhos/Fundação Oswaldo Cruz. Rio de Janeiro, Brasil. E-mail: ingrydventura@hotmail.com.

IIIEnfermeiro. Especialista em Enfermagem do Trabalho pela Universidade do Estado do Rio de Janeiro. Enfermeiro do Instituto Estadual de Hematologia Arthur de Siqueira Cavalcanti (Hemorio).Rio de Janeiro, Brasil. E-mail: feliperossone@hotmail.com.

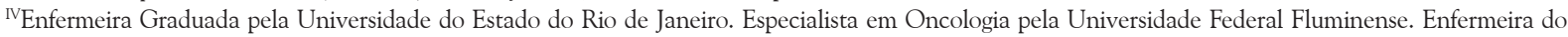
Hospital Municipal Dr. Nelson de Sá Earp. Rio de Janeiro, Brasil. E-mail: livia.delphim@gmail.com.

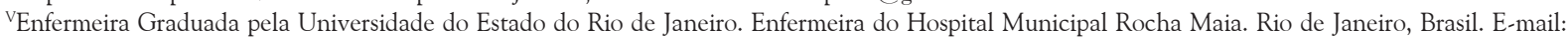
micheleteotonio94@msn.com.
} 
$\mathrm{O}$ estresse pode surgir quando um indivíduo se confronta com situações que o irritem, amedrontem, excitem, confundam ou mesmo aquelas que o fazem imensamente feliz, em virtude da interpretação que se dá ao evento desafiador ou em razão de estressores inerentemente negativos ${ }^{3}$.

Numerosos estudos associam estresse ao trabalho, o que evidencia a estreita relação entre os temas, e, portanto, a preocupação com o impacto negativo na saúde e no bem-estar dos trabalhadores, podendo gerar prejuízos para o funcionamento e efetividade das instituições ${ }^{4}$.

O hospital é uma instituição que se configura como um local de trabalho possivelmente estressante dadas suas características peculiares. "De maneira geral, é reconhecido como um ambiente insalubre, penoso e perigoso para os que ali trabalham"5:518. Inseridos neste cenário, encontram-se os profissionais de enfermagem cuja saúde tem merecido um cuidado especial $^{1}$.

Ao observar setores distintos dentro de um mesmo hospital, percebe-se que esses profissionais possuem diversas atribuições e responsabilidades específicas, dependendo do campo onde atuam. Por conseguinte, as cobranças internas e externas podem ter intensidades variáveis. Nesse contexto, surge o seguinte questionamento: quais equipes de enfermagem, atuantes em diferentes unidades de um hospital universitário, possuem maior nível de estresse?

Definiu-se como objetivo geral: discutir o nível de estresse oriundo do trabalho dos profissionais de enfermagem. E os seguintes objetivos específicos: comparar o nível de estresse das equipes de enfermagem atuantes em três setores de um Hospital Universitário; analisar a interferência dos fatores estressores oriundos do contexto de trabalho no nível de estresse das equipes de enfermagem.

Ressalta-se a importância deste estudo para a assistência de enfermagem, uma vez que está relacionada com a qualidade de vida do profissional, que quando comprometida, influencia na assistência prestada por ele. Em relação ao ensino, este estudo auxiliará na formação de profissionais mais críticos e preocupados com a sua própria saúde no ambiente laboral e que saibam identificar os fatores causadores de maiores níveis de estresse. $O$ resultado da pesquisa facilitará uma visão ampliada e maior compreensão do estresse e suas implicações no ambiente de trabalho, familiar e social.

\section{REVISÃO DE LITERATURA}

O estresse foi definido pela primeira vez como a resposta não específica do organismo diante de qualquer situação que ameace a homeostase do indivíduo, gerando a necessidade de mobilização para enfrentar o evento causador do desequilíbrio biopsicossocial ${ }^{6}$. Ele é a reação psicofisiológica que se caracteriza como o desequilíbrio entre o que é cobrado de uma pessoa pelo entorno social e a capacidade dela corresponder a tal cobrança?

O senso comum quase sempre pensa no estresse como algo ruim, o que nem sempre é assim, pois existem dois tipos: o chamado eustress utilizado para designar o bom estresse, que motiva o ser humano a atuar no mundo e que é natural e necessário na dinâmica da vida; já o distress está relacionado ao estresse ruim, caracterizado por desconforto moderado até o extremo, podendo gerar esgotamento físico e mental ${ }^{7}$.

$\bigcirc$ estresse pode ser dividido em quatro fases ${ }^{8}$ assim consideradas: alerta - fase na qual o indivíduo precisa de mais força e energia a fim responder àquilo que está exigindo dele um esforço maior ${ }^{9}$; resistência - é a constante busca pelo reequilíbrio, podendo gerar sensação como desgaste generalizado sem causa aparente e dificuldades na memória ${ }^{10}$; quase-exaustão - quando as defesas começam a se esgotar e a pessoa não consegue restabelecer a homeostase interior ${ }^{3}$. $\mathrm{O}$ aparecimento de algumas doenças, comum nessa fase, demonstra que a resistência já não está tão eficaz; e a quarta fase nomeada de exaustão - a resistência é totalmente quebrada e alguns sintomas semelhantes aos da fase de alarme voltam a aparecer, contudo, em maior magnitude. Apesar da intensa gravidade desta fase, ela não é, necessariamente, irreversível ${ }^{3}$.

As discussões sobre o estresse vêm adentrando o mundo do trabalho porque apesar de suprir diversas necessidades do homem e da família e ser uma das formas de busca pela auto-realização, o trabalho também pode ser fonte de adoecimento para o trabalhador ${ }^{1,11}$.

As atuais tendências na promoção da segurança no trabalho incluem não somente os riscos dos ambientes laborais, mas também os múltiplos fatores psicossociais inerentes ao ambiente de trabalho e a maneira como esses fatores influenciam na condição de saúde do trabalhador ${ }^{12}$. Esses riscos são os menos estudados, porém a desatenção com os mesmos não minimiza seu potencial de causar doenças ocupacionais ou relacionadas ao trabalho, como o estresse $\mathrm{e}^{13}$.

O estresse vivenciado no contexto ocupacional poderá repercutir sobre a qualidade e o nível de compromisso com o trabalho, além de influenciar o grau de satisfação pessoal e o próprio ambiente de trabalho, que incluem o relacionamento com colegas e supervisores. Trabalhadores doentes inevitavelmente diminuem a produtividade no trabalho, estão mais vulneráveis ao absenteísmo e aos afastamentos prolongados do ambiente laboral, o que poderá gerar prejuízo para as organizações ${ }^{13}$. $\mathrm{O}$ que se pode inferir que se instala um ciclo vicioso no qual o trabalhador e a instituição entram numa situação na qual ambos saem perdendo. Torna-se necessário pensar em estratégias para lidar com estas demandas do mundo do trabalho. 
A partir da análise de diversos estudos, foram encontrados alguns componentes conhecidos como ameaçadores à estabilidade do enfermeiro: a falta de um reconhecimento nítido entre o público em geral, de quem é o enfermeiro, a situação política na qual estamos imersos, com o achatamento dos salários, estreitamento do mercado de trabalho e o desemprego. Todos estes componentes são fatores agravantes aos profissionais que são obrigados a atuar em mais de um local de trabalho, exercendo uma carga horária mensal extremamente longa ${ }^{14}$.

Historicamente o enfermeiro tenta construir sua identidade profissional e obter reconhecimento. Dificuldades vivenciadas por e não são unicamente históricas, mas também situacionais. As atividades realizadas por estes profissionais os tornam especialmente suscetíveis ao fenômeno do estresse ocupacional. Baseados nestas afirmações, os estudos sobre o estresse na enfermagem não podem perder de vista esta dimensão ${ }^{14}$.

Em pesquisa realizada junto aos enfermeiros de centro cirúrgico elucidou-se a necessidade de realização de um trabalho comparativo entre as unidades distintas de atuação desses profissionais. Outros estudos de comparação foram realizados e tentouse definir um modelo de repercussão de estresse na atividade ocupacional da enfermagem. Todavia, os diversos referenciais teórico-práticos e metodológicos dificultam esse delineamento ${ }^{2}$.

Dessa forma, nota-se que a pesquisa sobre o estresse da equipe de enfermagem e sua correlação com o trabalho pode auxiliar na melhor compreensão e possível elucidação de alguns dos problemas enfrentados pela categoria. Intervenções e soluções serão possíveis quando houver uma melhor compreensão destes processos ${ }^{14}$.

\section{Metodologia}

O estudo é do tipo descritivo, com abordagem quantitativa. O campo de pesquisa foi um hospital universitário, situado no Município do Rio de Janeiro e suas unidades selecionadas foram o centro de tratamento intensivo (CTI) geral; duas enfermarias de clínica médica; e o ambulatório central. A escolha destas unidades justificou-se por se tratarem de cenários onde a assistência de enfermagem varia conforme a complexidade do cuidado prestado ao paciente atendido, o que viabilizou o alcance dos objetivos do estudo.

A população foi constituída por todos os profissionais da equipe de enfermagem, a saber: enfermeiros, residentes, técnicos e auxiliares de enfermagem que compunham o quadro funcional existente nos setores pesquisados. As amostras obtidas foram 19 sujeitos nas enfermarias, 25 no CTI e 41 no ambulatório central, totalizando 85 profissionais.
Foram utilizados como critérios de inclusão: profissionais atuantes há no mínimo 1 mês nas unidades selecionadas; todos os profissionais que estavam exercendo suas atividades laborais no período da coleta de dados do quadro efetivo ou de contrato temporário. Foram excluídos aqueles que se encontravam em regime de licença ou férias, que se recusaram a participar do estudo, e ainda os profissionais que estavam inacessíveis.

A pesquisa foi aprovada pelo Comitê de Ética em Pesquisa do Hospital, com o registro no 3005/2011, no dia 09 de agosto de 2011, por se tratar de um estudo envolvendo seres humanos. Ainda em consonância com a legislação, só participaram aqueles que assinaram o Termo de Consentimento Livre e Esclarecido (TCLE) contendo os objetivos e todas as informações a respeito da pesquisa, resguardando os preceitos éticos, bem como garantindo o direito dos trabalhadores em participar ou não do estudo sem prejuízos financeiros ou profissionais ${ }^{15}$.

A coleta de dados ocorreu entre os meses de agosto e setembro de 2011. Foram utilizados dois instrumentos para coleta de dados: Inventário de Sintomas de Stress para Adultos (ISSL) ${ }^{8}$; e outro elaborado pelos pesquisadores denominado Inventário de Avaliação de Fatores Estressores Subjetivos (IAFES) que foi embasado em dois instrumentos validados: o Job Stress Scale (JSS) ${ }^{16} \mathrm{e}$ o Inventário de Estresse para Enfermeiros (IEE) ${ }^{17}$.

O ISSL possibilita identificar a presença do estresse em adultos e a fase na qual a pessoa se encontra (alerta, resistência, quase-exaustão e exaustão) e a predominância de sintomas físicos e/ou psicológicos em cada fase. É composto de 56 itens sendo 37 de natureza somática, física e 19 de natureza psicológica. O instrumento constitui-se de três quadros: o primeiro é relativo aos sintomas apresentados nas últimas 24 horas - fase de alerta; o segundo identifica os sintomas experimentados na última semana - fases de resistência e quase-exaustão; e o terceiro se refere aos sintomas apresentados no último mês - fase de exaustão ${ }^{8}$.

O IAFES é composto de 19 itens que consistem em afirmativas sobre atitudes dos profissionais de enfermagem no ambiente laboral. É uma escala do tipo Likert na qual as respostas podem variar de nunca até sempre; como foram utilizados somente itens diretos, se o respondente concorda com as sentenças ele terá escores mais elevados e vice-versa. Foram considerados mais expostos aos fatores de riscos aqueles indivíduos que obtiveram os valores acima da média do somatório dos escores globais, e menos expostos os abaixo da média. O somatório dos escores pode variar de 20 até 100 .

Os dados oriundos do IAFES foram analisados através da distribuição de frequência dos escores obtidos por cada participante do estudo. Foram determinados pelos quartis e orientados pela média dos escores. A média foi 51,32 pontos para o nível de exposição aos fatores estressores no ambiente de trabalho, sendo o desvio padrão 10,98. 
Além disso, foi calculado o poder de discriminação dos itens através do teste t de Studant e o resultado evidenciou que todos os valores foram significativos para 19 itens $(p<0,05)$ e portanto foram aceitos. Somente o item 13 não alcançou valor significativo ( $\mathrm{p}$ $>0,05$ ) e então, foi rejeitado. Infere-se que este item, assim descrito: Faço plantões noturnos nesta unidade, fora rejeitado porque consiste em um fato e não em uma atitude. Ressalta-se que tal assertiva foi retirada.

A análise dos dados utilizou técnicas pertencentes à estatística descritiva que foi usada para descrever e sintetizar os dados, sendo realizados cálculos estatísticos para estimar os parâmetros ${ }^{18} \mathrm{com}$ apoio do software Microsoft Office Excel 2007.

\section{Resultados e Discussão}

A partir da proposta deste estudo, emergiram dados que possibilitaram ampliar a compreensão sobre a importância desta temática no campo da prática de enfermagem. De acordo com a análise do material coletado, as fases do estresse, nas quais os profissionais se encontram, estão representadas de acordo com os setores onde atuam, na Tabela 1.

Na avaliação do estresse, observa-se que do total de profissionais que participaram do estudo, a maioria, 48 (56,5\%), apresentava algum nível de estresse. Embora somente 4 (4,7\%) da amostra encontrem-se nas fases de quase exaustão e exaustão, este dado é relevante, pois a fase de quase exaustão dá início ao processo de adoecimento e os órgãos com maior vulnerabilidade passam a ser afetados pelo estresse ${ }^{8}$.

Comparando o nível de estresse entre as equipes de enfermagem atuantes nas unidades pesquisadas, constata-se que quase a metade, $42(49,4 \%)$, dos profis- sionais se encontra na fase de resistência. Dados estes que corroboram pesquisas anteriormente realizadas: uma que avaliou o nível de estresse de trabalhadores atuantes em uma unidade de saúde da família, cujos resultados evidenciaram que, em sua maioria, se encontravam na fase de resistência ${ }^{19}$. Outra, cujo objetivo foi avaliar o grau de estresse em graduandos de enfermagem a partir do inventário de Lipp, evidenciou que a maioria dos estudantes, correspondendo a 146 (68,5\%), apresenta algum grau de estresse. Destes, 115 (54\%) encontram-se na fase II, resistência, sugerindo que os graduandos, possivelmente, precisam de grande esforço para gerenciar o estresse ${ }^{20}$.

Elucidando os dados obtidos, estudos constataram que o estresse influencia de maneira negativa o desempenho das atividades laborais dos profissionais de enfermagem ${ }^{1}$. Isto pode ser afirmado, uma vez que esses trabalhadores

se sentem sobrecarregados física e emocionalmente, desestimulados, com irritações frequentes, malhumorados, frustrados, com dores musculares; sinais e sintomas característicos do estresse ${ }^{1: 701}$.

Outro aspecto relevante, em se tratando da fase de resistência, é pensar que ela se caracteriza pelo aumento da produção de cortisol levando a pessoa à maior vulnerabilidade às doenças ${ }^{8}$. É o momento no qual pode surgir a somatização.

A relação entre o nível de estresse com os cargos exercidos pelos participantes da pesquisa aponta que, dos técnicos de enfermagem, a maioria - 24 (51,1\%) - não apresenta estresse. No entanto, um parte significativa, $18(38,3 \%)$, está na fase de resistência, seguida de $3(6,4 \%)$ na fase de quase-exaustão. Dados estes apresentados na Tabela 2.

TABELA 1: Nível de estresse dos profissionais de enfermagem por unidades. Rio de Janeiro, 2011.

\begin{tabular}{lcccccccc}
\hline Fases de estresse por & \multicolumn{2}{c}{ Enfermarias } & \multicolumn{2}{c}{ CTI } & \multicolumn{2}{c}{ Ambulatório } & \multicolumn{2}{c}{ TOTAL } \\
\cline { 2 - 9 } setores & $\mathrm{f}$ & $\%$ & $\mathrm{f}$ & $\%$ & $\mathrm{f}$ & $\%$ & $\mathrm{f}$ & $\%$ \\
\hline Sem estresse & 9 & 47,4 & 11 & 44 & 17 & 41,5 & 37 & 43,5 \\
Fase de Alerta & - & - & - & - & 2 & 4,9 & 2 & 2,4 \\
Fase de Resistência & 10 & 52,6 & 13 & 52 & 19 & 46,3 & 42 & 49,4 \\
Fase de Quase Exaustão & - & - & - & - & 3 & 7,3 & 3 & 3,5 \\
Fase de Exaustão & - & - & 1 & 4 & - & - & 1 & 1,2 \\
\hline Total & $\mathbf{1 9}$ & $\mathbf{1 0 0}$ & $\mathbf{2 5}$ & $\mathbf{1 0 0}$ & $\mathbf{4 1}$ & $\mathbf{1 0 0}$ & $\mathbf{8 5}$ & $\mathbf{1 0 0}$ \\
\hline
\end{tabular}

TABELA 2: Nível de estresse por cargo exercido. Rio de janeiro, 2011.

\begin{tabular}{lcccccccccc}
\hline \multirow{2}{*}{$\begin{array}{l}\text { Nível Estresse por } \\
\text { cargo exercido }\end{array}$} & \multicolumn{2}{c}{ Enfermeiro } & \multicolumn{2}{c}{ Residente } & \multicolumn{2}{c}{ Técnico } & \multicolumn{2}{c}{ Auxiliar } & \multicolumn{2}{c}{ TOTAL } \\
\cline { 2 - 14 } & $\mathrm{f}$ & $\%$ & $\mathrm{f}$ & $\%$ & $\mathrm{f}$ & $\%$ & $\mathrm{f}$ & $\%$ & $\mathrm{f}$ & $\%$ \\
\hline Sem estresse & 10 & 35,7 & 3 & 37,5 & 24 & 51,1 & - & - & 37 & 43,5 \\
Alerta & 1 & 3,6 & - & - & 1 & 2,1 & - & - & 2 & 2,4 \\
Resistência & 17 & 60,7 & 5 & 62,5 & 18 & 38,3 & 2 & 100 & 42 & 49,4 \\
Quase-exaustão & - & - & - & - & 3 & 6,4 & - & - & 3 & 3,5 \\
Exaustão & - & - & - & - & 1 & 2.1 & - & - & 1 & 1,2 \\
\hline Total & $\mathbf{2 8}$ & 100 & $\mathbf{8}$ & $\mathbf{1 0 0}$ & $\mathbf{4 7}$ & 100 & $\mathbf{2}$ & 100 & $\mathbf{8 5}$ & $\mathbf{1 0 0}$ \\
\hline
\end{tabular}


Somando-se os técnicos de enfermagem situados nas diferentes fases, fica evidenciado elevado número sob efeito de estresse, correspondendo a 22 (46,8\%). Quanto aos residentes de enfermagem, a maioria, $5(62,5 \%)$, encontra-se na fase de resistência. Dos enfermeiros pesquisados, verificou-se que 10 (35,7\%) não revelam nenhum nível de estresse, 1 (3,6\%) está na fase de alerta e a maioria, totalizando $17(60,7 \%)$, na fase de resistência. Ver Tabela 2.

Os dados evidenciam que a presença do estresse na fase de resistência não está diretamente relacionada ao cargo ocupado pelo profissional, visto que das quatro categorias pesquisadas, todas apresentam parte significativa de indivíduos nessa fase.

A partir dos resultados encontrados neste trabalho e em pesquisas anteriores, percebe-se que, independente do cargo, todos os profissionais de enfermagem experimentam situações promotoras de estresse similares. Alguns eventos vivenciados por esses profissionais englobam: o sentimento de impotência diante da dor e morte; a cobrança exagerada das chefias, dos outros trabalhadores e pacientes; e a falta de tempo para lazer e descanso. Em resposta a estes acontecimentos, a idealização da profissão surge como uma forma de enfrentar os fatores estressores e evitar sofrimento ${ }^{5}$.

A classificação da exposição dos profissionais ficou determinada pelo primeiro quartil (menor ou igual a 45), segundo quartil (46 a 50), terceiro quartil (51 a 55) e quarto quartil (maior ou igual a 56). Portanto, a análise dos resultados destacou: 31 (36,5\%) participantes não se expõem aos fatores estressores; 13 (15,3\%) foram considerados com média exposição; 19 $(22,3 \%)$ com média-alta exposição e 22 (25,9\%) com alta exposição, como mostra a Tabela 3.

Sobre o nível de exposição aos fatores estressores dos profissionais por unidades de trabalho, a maioria, 54 (63,5\%), encontra-se em média, média-alta ou alta exposição aos fatores estressores oriundos do trabalho. As enfermarias apresentaram o maior percentual de profissionais em alta exposição, totalizando 8 (42,1\%). Ver Tabela 3.

Há uma consonância entre estes dados e os apresentados na Tabela 1, uma vez que os partici- pantes lotados em todas as unidades estão na fase de resistência, quase-exaustão e exaustão - 46 (54,1\%). A partir do exposto, pode-se inferir que os fatores estressores presentes na atividade laboral podem ter influência direta nos níveis alterados de estresse dos profissionais, em especial das enfermarias.

Entre os participantes deste estudo estão os residentes de enfermagem. Nesse sentido, pesquisa realizada com esses profissionais apontou que todos os $16(100 \%)$ participantes se sentem estressados por conta da pressão em relação à carga horária exaustiva de trabalho. Em se tratando de profissionais em proceso de formação, é importante levar em consideração a inexperiência para o enfrentamento de situações novas e imprevistas ${ }^{21}$.

Para que não cheguem aos extremos de adoecimento e mantenham a sua saúde protegida, é preciso que estes trabalhadores desenvolvam, frente aos fatores estressores vivenciados, mecanismos de enfrentamento mais eficazes a fim de amenizar o impacto psicológico e somático do estresse e, assim, aumentar em frequência e intensidade os estados emocionais positivos, que interferem direta e indiretamente na saúde física desses profissionais ${ }^{22}$.

Autores afirmam ser importante o investimento em programas de manejo de estresse ocupacional, a fim de intervir nesses grupos de trabalhadores, promovendo segurança e saúde, mantendo os níveis ideais de produtividade e também reduzindo os gastos das organizações com possíveis adoecimentos ${ }^{11}$.

Estudos anteriores mostram algumas estratégias de enfrentamento do estresse por profissionais de saúde $^{12,23,24}$. Um estudo sobre estratégias de coping utilizadas por enfermeiros que atuam em oncologia obtiveram como mais relevantes as estratégias focadas na emoção, essencialmente a reavaliação positiva, em que o indivíduo revê os acontecimentos a fim de encontrar aspectos positivos na vivência e falar coisas para si próprio com intuito de amenizar a situação estressora. Outra estratégia utilizada foi o coping focado no problema, visando à sua resolução ${ }^{23}$.

Ainda sobre estratégias de enfrentamento utilizadas, um estudo realizado junto à equipe de enfermagem, que atua no pronto atendimento de um centro

TABELA 3: Nível de exposição aos fatores estressores por unidade de trabalho dos participantes. Rio de Janeiro, 2011.

\begin{tabular}{lcccccccc}
\hline Nível de exposição por & \multicolumn{2}{c}{ Enfermarias } & \multicolumn{2}{c}{ CTI } & \multicolumn{2}{c}{ Ambulatório } & \multicolumn{2}{c}{ TOTAL } \\
\cline { 2 - 9 } unidade & $\mathbf{f}$ & $\%$ & $\mathbf{f}$ & $\%$ & $\mathbf{f}$ & $\%$ & $\mathbf{f}$ & $\%$ \\
\hline Sem Exposição & 6 & 31,6 & 10 & 40 & 15 & 36,6 & 31 & 36,5 \\
Média Exposição & 2 & 10,5 & 4 & 16 & 7 & 17,1 & 13 & 15,3 \\
Média-alta Exposição & 3 & 15,8 & 8 & 32 & 8 & 19,5 & 19 & 22,3 \\
Alta Exposição & 8 & 42,1 & 3 & 12 & 11 & 26,8 & 22 & 25,9 \\
\hline TOTAL & $\mathbf{1 9}$ & $\mathbf{1 0 0}$ & $\mathbf{2 5}$ & $\mathbf{1 0 0}$ & $\mathbf{4 1}$ & $\mathbf{1 0 0}$ & $\mathbf{8 5}$ & $\mathbf{1 0 0}$ \\
\hline
\end{tabular}


de saúde escola, aponta estratégias de evitamento, confronto direto (buscar informações, falar sobre o assunto e negociar alternativas) e indireto (ligadas às atividades religiosas e à prática esportiva) frente ao estresse $^{22}$. Observou-se também uma estratégia aplicada pelo serviço, comum a todas as equipes, que englobam reuniões semanais com exposição das situações problemas e/ou necessidades da população, assim como, soluções para o cotidiano de seu trabalho ${ }^{12}$.

Embora os profissionais se utilizem das diversas estratégias de enfrentamento diante dos fatores estressores ocupacionais, é necessário um trabalho que vise identificar e minimizar diretamente os causadores de estresse no ambiente laboral, pois estas estratégias de coping, mesmo que proporcionem um relaxamento temporário, não modificam a situação geradora de estresse ${ }^{22}$.

\section{Conclusão}

Para a realização desta pesquisa, algumas limitações foram enfrentadas, podendo-se destacar de maneira especial a dificuldade de encontrar os profissionais em momentos de disponibilidade para responder aos questionários, visto que muitas vezes estes se encontravam atarefados. Mesmo diante disso, foi possível realizar um trabalho de qualidade e com resultados que merecem atenção, pois dizem respeito à saúde do trabalhador.

A partir deste estudo foi possível constatar que as equipes de enfermagem, atuantes nas diferentes unidades pesquisadas, não apresentaram diferenças quanto ao nível de estresse. No entanto, evidenciouse que os fatores estressores oriundos do contexto de trabalho interferem no nível de estresse das equipes.

Apesar de não representarem um percentual significativo, ressalta-se que alguns participantes encontram-se nas fases mais avançadas do estresse, além da resistência. Diante desse contexto, percebe-se a necessidade se pensar em estratégias de intervenção que possam reduzir os fatores estressores laborais, mas também seus efeitos sobre os trabalhadores.

Algumas situações no mundo do trabalho são difíceis de serem modificadas em curto prazo, quiçá em médio prazo, então nesse tempo, é preciso cuidar dos trabalhadores. Para tal, sugere-se capacitação em gerenciamento de estresse e de conflitos, capacitação em habilidades sociais e criação de grupos de suporte ao trabalhador. Estas estratégias podem levar ao fortalecimento dos recursos internos e ajudá-los a aprender a gerenciar o estresse, mudando a maneira de perceber e de lidar com a situação estressora apresentada.

\section{REFERÊNCIAS}

1.Hanzelmann RS, Passos JP. Imagens e representações da enfermagem acerca do stress e sua influência na atividade laboral. Rev esc enferm USP. 2010; 44: 694-701.
2.Bianchi ERF. Enfermeiro hospitalar e o stress. Rev esc enferm USP. 2000; 34: 390-4

3.Lipp MEN. O modelo quadrifásico do stress. In: Lipp MEN, organizadora. Mecanismos neuropsicofisiológicos do stress: teoria e aplicações clínicas. São Paulo: Casa do Psicólogo; 2003. p. 17-21.

4.Hökerberg YHM. Demanda e controle no trabalho e hipertensão arterial: validade dimensional e meta-análise [tese de doutorado]. Rio de Janeiro: Universidade do Estado do Rio de Janeiro; 2010.

5.Elias MA, Navarro VL. A relação entre o trabalho, a saúde e as condições de vida: negatividade e positividade no trabalho das profissionais de enfermagem de um hospital escola. Rev Latino-Am Enfermagem. [Internet]. 2006 [citado em 25 mar 2014];14:517-25. Disponível em: http://www.facenf.uerj.br/v19n1/v19n1a23.pdf 6.Selye H. Stress: a tensão da vida. São Paulo: Ibrasa; 1959. 7.Ribeiro MAP, Ribeiro LTF. Estresse: conhecer para superar. Petrópolis (RJ): Vozes; 2005.

8.Lipp MEN. Inventário de sintomas de stress para adultos de Lipp (ISSL). São Paulo: Casa do Psicólogo; 2000. 9.Margis R, Picon P, Cosner AF, Silveira ROS. Relação entre estressores, estresse e ansiedade. Rev Psiquiat. [Internet] 2003 [citado em 21 abr 2014];25 (1):65-74. Disponível em: http://www.scielo.br/pdf/rprs/v25s1/a08v25s1.pdf.

10.Lipp MEN, Malagris LEN. Manejo do estresse. In: Range B, organizador. Psicoterapia comportamental e cognitiva: pesquisa, aplicações e problemas. Campinas (SP): Editorial Psy; 1995. p. 17-21.

11.Murta SG, Tróccoli BT. Avaliação de intervenção em estresse ocupacional. Psicol Teor Pesq. 2004; 20 (1):39-47. 12. Camelo SHH, Angerami ELS. Riscos psicossociais no trabalho que podem levar ao estresse: uma análise da literatura. Ciênc Cuid Saúd. [Internet]. 2008 [citado em 24 mai 2014];7:232-40. Disponível em: http://periodicos.uem.br/ojs/index.php/CiencCuidSaude/article/ view/5010/3246

13. Guimarães LAM. Fatores psicossociais de risco no trabalho. In: Anais do $2^{\circ}$ Congresso Internacional sobre Saúde Mental no Trabalho: 2006; Goiânia Brasil. Goiânia (GO): [s.e.]; 2006. p. 99-110.

14.Stacciarini JMR, Tróccoli BT. O estresse na atividade ocupacional do enfermeiro. Rev Latino-Am Enfermagem. 2001; 9(2): 17-25.

15.Ministério da Saúde (Br). Normas de pesquisa envolvendo seres humanos. Brasília (DF): Ministério da Saúde; 1996.

16.Alves MGM, Chor D, Faerstein E, Lopes CS, Werneck GL. Versão resumida da "job stress scale": adaptação para o português. Rev Saude Publica. [Internet] 2004; [citado em 10 abr 2014];38:164-71. Disponível em: http://www. scielosp.org/scielo.php?script $=$ sci_arttext $\&$ pid $=$ S0034 $89102004000200003 \& \operatorname{lng}=\mathrm{en} \& \bar{n} r \mathrm{~m}=$ iso

17.Stacciarini JMR, Trócolli BT. Instrumento para mensurar o estresse ocupacional: inventário de estresse em enfermeiros (IEE). Rev Latino-Am Enfermagem [Internet]. 2000 [citado em 18 abr 2014]; 8(6):40-9. Disponível em: http://www.scielo.br/pdf/rlae/v8n6/12347.pdf.

18.Polit D, Beck CT, Hungler B. Fundamentos de pesquisa em enfermagem. Porto Alegre (RS): Artmed; 2004. 
19. Camelo SHH, Angerami ELS. Sintomas de estresse nos trabalhadores atuantes em cinco núcleos de saúde da família. Rev Latino-Am Enfermagem. [Internet] 2004 [citado em 05 nov 2014];12: 14-21. Disponível em: http:// www.scielo.br/scielo.php?script $=$ sci_arttext\&pid $=$ S0104$11692004000100003 \& \operatorname{lng}=$ en\& $\&$ rm $=$ iso

20.Kestenberg CCF, Silva AV, Fabri JMG, Silva NABS, Rosa BMS, Branco LM. Estresse em graduando de enfermagem: técnicas de relaxamento para lidar com fatores estressores. Rev Interagir. 2014; (17): 37-43.

21.Oliveira EB, Souza NVM, Chagas SCS, Lima LSV, CRA. Esforço e recompensa no trabalho do enfermeiro residente em unidades especializadas, Rev enferm UERJ [Internet] 2013 [citado em 28 jan 2015]; 21:173-8. Disponível em: http://www.facenf.uerj.br/v19n1/v19n1a23.pdf
22.Steffy BD, Jones JW, Noe AW. The impact of health habits and life-style on the stressor-strain relationship: an evaluation of three industries. Journal of Occupational Psychology. 1990; 63: 217-29.

23.Calderero ARL, Miasso AI.; Corradi-Webster CM. Estresse e estratégias de enfrentamento em uma equipe de enfermagem de pronto atendimento. Rev Eletr Enf. [Internet]. 2008 [citado em 13 abr 2014];10(1):51-62. Disponível em: http://www.fen.ufg.br/revista/v10/n1/ v10n1a05.htm

24.Rodrigues AB, Chaves EC. Fatores estressantes e estratégias de coping dos enfermeiros atuantes em oncologia. Rev Latino-Am Enfermagem. [Internet] 2008 [citado em 23 mai 2014];16:24-8. Disponível em: http:// www.scielo.br/pdf/rlae/v16n1/pt_03.pdf. 